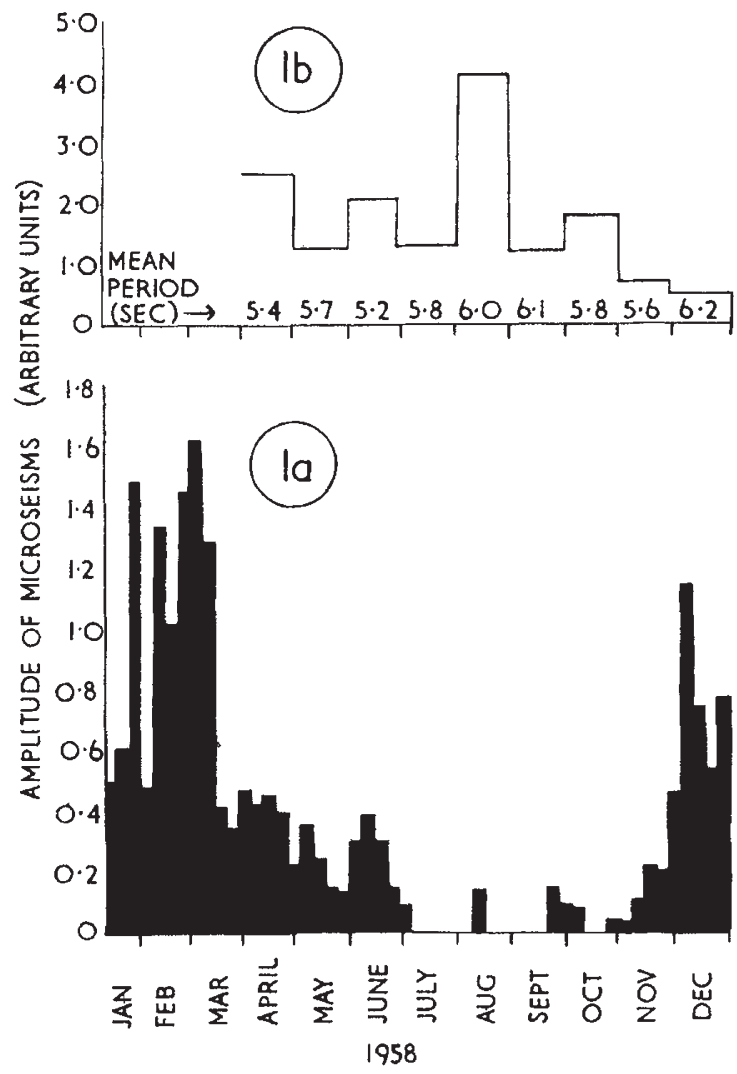

Fig. 1. (a) Weekly mean amplitudes of microseismic activity on short-period vertical record, Scott Base, 1958. Based on five typical groups at $00,06,12$ and $18 \mathrm{hr}$. daily, (b) Monthly mean amplitudes of long-period microseismic activity on long-period north-south record, Scott Base, 1958. Based on five typical mean periods are given (in seconds) at base of histogram

activity are widespread in the literature but the mechanism of generation and propagation is still unresolved and theories pertaining to it increase ${ }^{2}$. It may be possible to use a knowledge of the pack ice morphology (amplified by aerial photography) as a constraining factor in the examination of these theories.

Microseisms of the 4-10 sec. type appear throughout the year on the north-south component with the 10 sec. galvanometer. By taking the 6-hourly microseismic characteristics on every seventh day and computing monthly means, the histogram in Fig. $1 b$ has been derived. No satisfactory values for the months of January, February and March 1958 can readily be derived for these longer-period microseisms because of interference by shorter-period activity, which follows the same seasonal pattern as that on the short period vertical record. Mean periods (in seconds) of all observations are recorded for each month.

There may be a seasonal variation of the 4-10 sec. microseisms with a minimum in the summer; the results presented are certainly insufficient to establish this. But the major point of interest is that these microseisms persist undiminished throughout the winter. Their origins probably lie in the cyclones over the oceans surrounding the antarctic continent; and it seems impossible that wave or surf action at the coast could play any part in their generation or transmission.
This communication gives a broad picture of the principal events. There are many details of interest and these will be studied extensively in the future.

Lieut. F. Faggioni, of the Italian Navy, was the seismologist at Scott Base during 1958.

T. HATHERTON R. H. ORR

Geophysics Division,

Department of Scientific and

Industrial Research, Wellington,

New Zealand. April 21.

${ }^{1}$ Donn, W. L., Science, 119, 56 (1954).

${ }^{2}$ Donn, W. L., Trans. Amer. Geophys. Union, 38, 358 (1957).

\section{A Possible Experimental Test of Einstein's Second Postulate}

As is well known, Einstein's special theory of relativity rests on two postulates : (1) the postulate of relativity; (2) the postulate of constant light velocity, which says" "that light is always propagated in empty space with a definite velocity $c$ which is independent of the state of motion of the emitting. body". For the first postulate there is much experimental support; for the second, none. It is not generally appreciated that the experiments often held to confirm the kinematical part of Einstein's theory are, in fact, evidence that the MaxwellLorentz electromagnetic equations are invariant to the Lorentz transformation, since the forces and velocities concerned are determined from those equations and not by direct measurement.

In view of difficulties which have recently arisen, it is highly desirable to test the kinematics of the theory. It is often said that an experimental test of the second postulate is impossible since the clocks used would have to be synchronized by assuming its truth, but this is not so. If beams of light were emitted from the same point at the same instant from two relatively moving bodies, the postulate could be confirmed or otherwise by observing whether they arrived at a distant point together or successively; it would not be necessary to make a precise measurement of the times of travel. The frequencies need not be the same (the Doppler effect would make this unlikely in any event) provided the effect of the intervening air on the velocities was taken into account.

It might not be beyond the power of modern techniques to make this test by a purely terrestrial experiment, in which the motion of the sources remains effectively constant during the passage of the light. Forty years ago Majorena ${ }^{2}$ attained a velocity sufficient to test the indifference of the Michelson-Morley experiment to the velocity of the source, but this, of course, only confirmed the first postulate. My purpose here is to urge the importance of making this test if possible, in view of the very great amount of theory which at present rests on an assumption.

\section{Herbert Dingle}

104 Downs Court Road, Purley,

Surrey. 'Ann. Phys., 17, 891 (1905); English translation in "The Principle

${ }^{2}$ Phil. Mag., 37, 145 (1919). 